\title{
Multiple hepatic lesions in a case of isolated hepatic tuberculosis simulating metastases on ${ }^{18 F-F D G ~ P E T / C T ~ i m a g i n g ~}$
}

Sellam Karunanithi ${ }^{1}$, Punit Sharma1, Tarun Kumar Jain ${ }^{1}$, Maneesh Kumar Vijay², Rakesh Kumar ${ }^{1}$

${ }^{1}$ Department of Nuclear Medicine, All India Institute of Medical Sciences, New Delhi, India

2Department of Pathology, All India Institute of Medical Sciences, New Delhi, India

[Received 7 I 2014; Accepted 7 VII 2014]

\begin{abstract}
Hepatic tuberculosis is an unusual form of extrapulmonary tuberculosis and constitutes less than $1 \%$ of all cases of tuberculosis. Imaging studies for hepatic tuberculosis are nonspecific and mimic primary or metastatic carcinoma. Here we present ${ }^{18} \mathrm{~F}-\mathrm{FDG}$ $\mathrm{PET} / \mathrm{CT}$ images of a 25-year-old male patient with isolated hepatic tuberculosis.
\end{abstract}

KEY words: liver, tuberculosis, ${ }^{18}$ F-FDG, PET/CT

Nuclear Med Rev 2014; 17, 2: 108-109

\section{Introduction}

Hepatic tuberculosis is an unusual form of extrapulmonary tuberculosis and constitutes less than $1 \%$ of all cases of tuberculosis [1]. In recent years, abdominal tuberculosis has become rare in many countries even where pulmonary tuberculosis still continues to be endemic. Imaging studies for hepatic tuberculosis are nonspecific and mimic primary or metastatic carcinoma. Here we present ${ }^{18} \mathrm{~F}$-FDG PET/CT images of a 25 -year-old male patient with isolated hepatic tuberculosis.

\section{Case report}

A 25-year-old male patient presented with anorexia and weight loss of two months duration. On physical examination no abnormality was detected. His serum alanine aminotransferase (150 U/L, normal range: 1-40 U/L) and aspartate aminotransferase (60 U/L, normal range: $1-40 \mathrm{U} / \mathrm{L}$ ) were elevated. An ultrasonogram (USG) of the abdomen was performed which revealed a small sized right lobe of liver with left lobe hypertrophy. Also noted were few hypoechoic parenchymal lesions along with minimal bilobar intra hepatic biliary radical dilatation. The patient was referred for ${ }^{18} \mathrm{~F}-\mathrm{FDG}$

Correspondence to: Sellam Karunanithi, MD

Department of Nuclear Medicine, All India Institute of Medical Sciences,

New Delhi, India

Phone: 9873754137

E-mail: drsellam84@yahoo.co.in
PET/CT to identify the nature of the hepatic lesions. Whole body ${ }^{18} \mathrm{~F}-\mathrm{FDG} \mathrm{PET} / \mathrm{CT}$ was performed which revealed multiple ${ }^{18} \mathrm{~F}-\mathrm{FDG}$ avid (SUVmax - 10.6) hypodense lesions involving both lobes of liver with the largest lesion measuring 19*15 mm in size (Figure 1). No other focus of abnormal ${ }^{18} \mathrm{~F}-\mathrm{FDG}$ uptake was noted in the rest of the body. A differential diagnosis of liver metastases versus infective lesions was considered. The patient then underwent USG-guided aspirate from one of the liver lesion which revealed epithelioid cell granuloma and Langhans giant cells in the background of hepatocytes, suggestive of hepatic tuberculosis (Figure 2). A final diagnosis of isolated hepatic tuberculosis was made and the patient was started on antitubercular drugs.

\section{Discussion}

Levine [2] classified hepatic tuberculosis as miliary tuberculosis, pulmonary tuberculosis with hepatic involvement, primary liver tuberculosis, focal tuberculoma or abscess, or tuberculous cholangitis. It has been reported that size of the hepatic nodules greater than $2 \mathrm{~mm}$ are generally diagnosed as macronodular hepatic tuberculosis [3]. Hepatic tuberculosis is usually misrecognized as a primary or metastatic hepatic tumor. Isolated hepatic tuberculosis without pulmonary or bowel involvement is a diagnostic challenge and can cause considerable morbidity. Imaging studies for hepatic tuberculosis are nonspecific and usually mimic primary or metastatic carcinoma [4, 5]. Hence, hepatic tuberculosis should be considered in the differential diagnosis in a patient presenting with liver nodules, and histopathologic confirmation is necessary to direct subsequent management. Clinical and imaging features of 

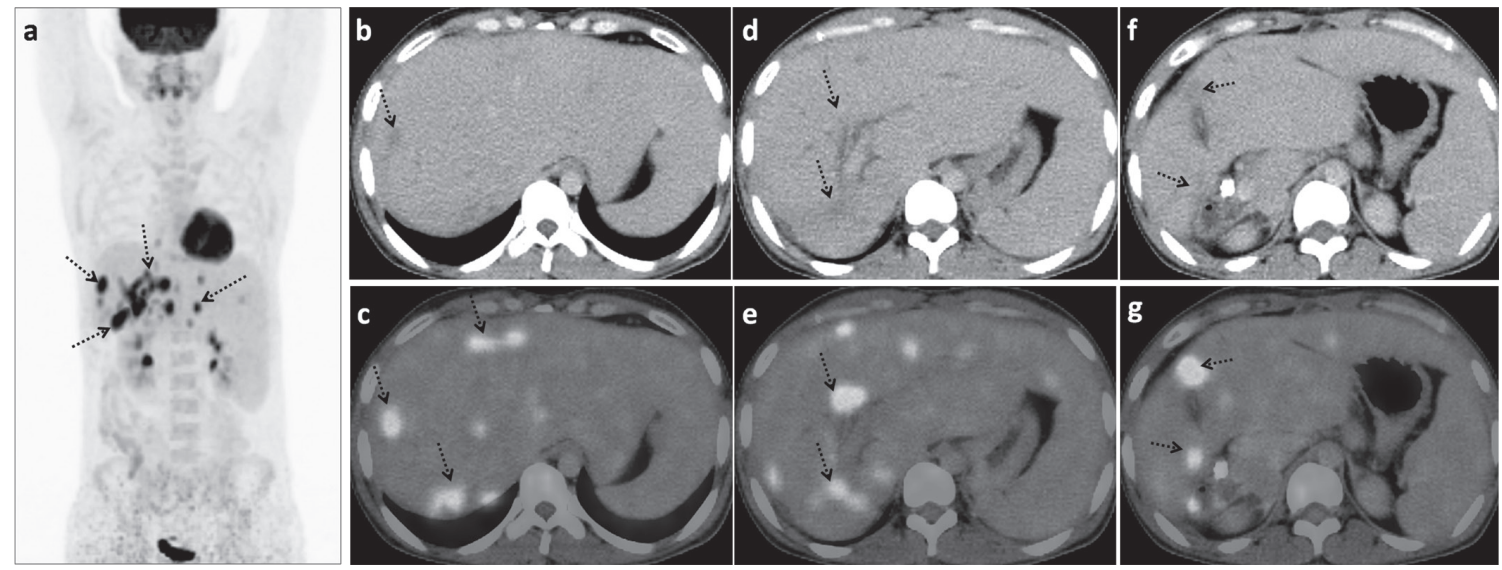

Figure 1. ${ }^{18} \mathrm{~F}-\mathrm{FDG}$ PET-CT maximum intensity projection (A) and trans-axial sections (B-G) images revealed multiple ${ }^{18} \mathrm{~F}-\mathrm{FDG}$ avid (SUVmax 10.6) hypodense lesions involving both lobes of liver (arrows) with the largest lesion measuring $19 \star 15 \mathrm{~mm}$ in size. No other focus of abnormal ${ }^{18} \mathrm{~F}-\mathrm{FDG}$ uptake was noted in the rest of the body $(\mathbf{A})$
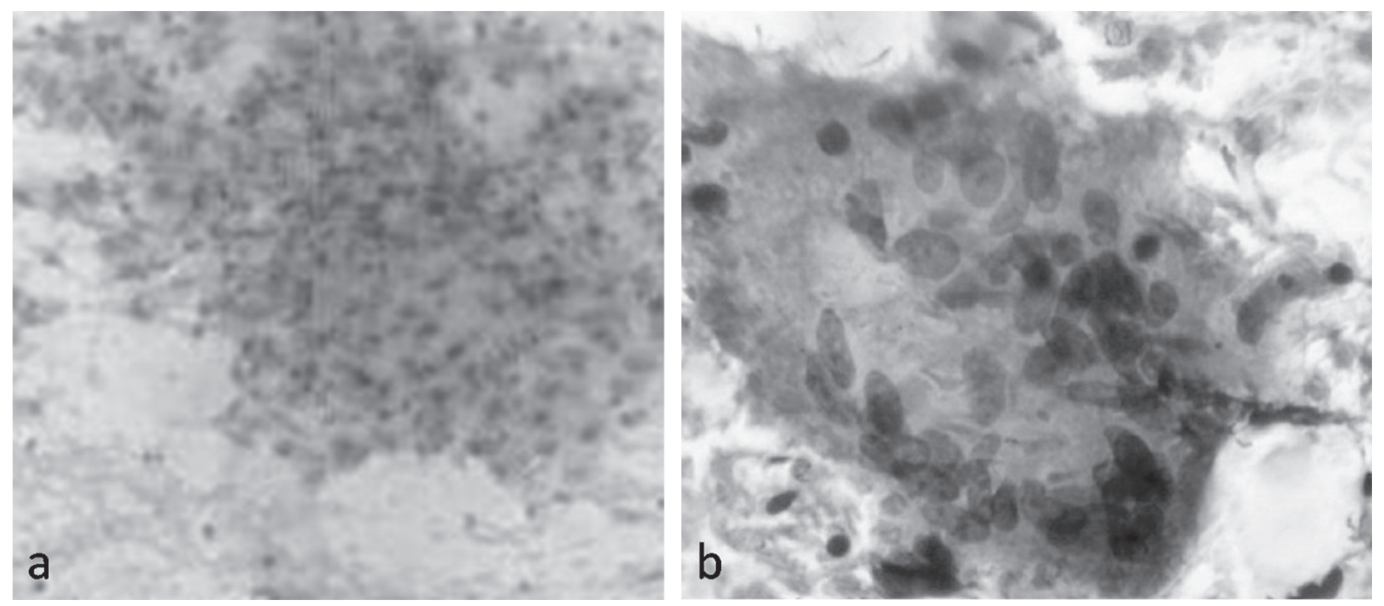

Figure 2. Ultrasound guided aspirate from liver: Papanicolaou (A) stained smear $(100 \times)$ and Giemsa (B) stained smear $(200 \times)$ showed epithelioid cell granuloma and Langhans giant cells in the background of hepatocytes suggestive of hepatic tuberculosis

hepatic tuberculosis are not specific and only a percutaneous biopsy of the lesion provides a definite diagnosis [6]. Histologically, the presence of a caseating granuloma is diagnostic for hepatic tuberculosis [7]. Cultures of the diseased liver are usually negative.

Quadruple therapy (isoniazid, rifampin, pyrazinamide and ethambutol) for at least 1 year is recommended because of the increasing incidence of drug-resistant tuberculosis [1]. Although findings of hepatic tuberculoma (single lesion) has been documented in ${ }^{18} \mathrm{~F}$-FDG PET/CT imaging [5], multiple hepatic lesions simulating metastatic lesions on ${ }^{18} \mathrm{~F}$-FDG PET/CT has not been documented. ${ }^{18} \mathrm{~F}-\mathrm{FDG}$ PET/CT has been found to be helpful for the differential diagnosis of extra-pulmonary tuberculosis from malignancy, but overlap occur [8]. Although rare, tuberculosis should also be considered in the differential diagnosis for ${ }^{18} \mathrm{~F}-\mathrm{FDG}$ avid multiple nodules in the liver, along with lymphoproliferative diseases and metastatic lesions, especially in young patients.

\section{References}

1. Mert A, Ozaras R, Tabak F, Ozturk R, Bilir M. Localized hepatic tuberculosis. Eur J Intern Med 2003; 14: 511-512.

2. Levine C. Primary macronodular hepatic tuberculosis: US and CT appearances. Gastrointest Radiol 1990; 15: 307-930.

3. Sarkar S, Saha K, Das CS. Isolated tuberculous liver abscess in a patient with asymptomatic stage I sarcoidosis. Respir Care 2010; 55: 1751-1753.

4. Varela M, Fernández J, Navasa M, Bruix J. Pseudotumoral hepatic tuberculosis. J Hepatol 2003; 39: 654.

5. Wang YT, Lu F, Zhu F, Qian ZB, Xu YP, Meng T. Primary hepatic tuberculoma appears similar to hepatic malignancy on F-18 FDG PET/CT. Clin Nucl Med 2009; 34: 528-529.

6. Tan TC, Cheung AY, Wan WY, Chen TC. Tuberculoma of the liver presenting as a hyperechoic mass on ultrasound. Br J Radiol 1997; 70: 1293-1295.

7. Alvarez SZ. Hepatobiliary tuberculosis. J Gastroenterol Hepatol 1998; 13: 833-839.

8. Liu GC, Gao S, Cai L, Chen QS. Characteristics of extrapulmonary tuberculosis in (18)F-2-fluoro-2-deoxy-D-glucose positron emission tomography-computed tomography: experience from 39 cases. Zhonghua Jie He He Hu Xi Za Zhi 2012; 35: 184-188. 